\title{
Quantum Chemical Model of Technetium Carbide
}

\author{
Yu. V. Plekhanov* and K. E. German \\ 119991, A.N.Frumkin Institute of Physical Chemistry and Electrochemistry, Russian Academy of Sciences, 31, \\ Leninskii pr., Moscow, Russia
}

Received: July 31, 2005; In Final Form: September 30, 2005

The discrete-variational (DV- $\left.\mathrm{X}_{\alpha}\right)$ method and Mulliken bond overlap population analyses were performed to study the electronic structure of technetium carbide and clarify the chemical formula $\mathrm{Tc}_{6} \mathrm{C}$ proposed for this compound.

Technetium carbide is an interesting alternative material of metallic technetium with respect to target fabrication for transmutation of radioactive ${ }^{99} \mathrm{Tc}$. Its properties, although briefly investigated, ${ }^{1}$ suppose that the targets are somewhat easier to fabricate, they should be quite stable under the neutron flux and could help to increase the transmutation rate by supplementary neutron thermalization inside the targets due to carbon atoms present. Also, according to the instability of ruthenium carbide, the partially transmuted targets should be less noble compared to Tc-Ru alloy and should be easily dissolved ${ }^{2}$ for the further Tc/Ru separation. This compound presents all properties of a metal phase and because of its C-deficient structure it could be considered as a quasi-equilibrium metallic phase stabilized with C-impurity, in contrast to the well-known hexagonal structure of Tc metal.

To clarify a proposed chemical formula ${ }^{1}$ for $\mathrm{Tc}_{6} \mathrm{C}$, quantum chemical calculations based on the hypothetical structure is required. As reported in Reference 3 for a metallic Tc, the calculations were made by non-empirical electron density functional in DV-X $\mathrm{X}_{\alpha}$ approximation by the DVSCAT code. ${ }^{4}$ We used the full electronic basis of numerical $1 s-5 p$ atomic orbitals for Tc and $1 s-2 p$ functions for $\mathrm{C}$. The electron exchange was accounted for by the Slater procedure ${ }^{5}$ with the parameter $\alpha=$ 0.7 for all atoms. The individual contributions of the atomic orbitals were estimated by the Mulliken population analysis. ${ }^{6}$ The number of integrating points for numerical estimation of matrix elements were $500 N_{a}$, where $N_{a}$ is the number of atoms in model clusters.

As a starting model of metallic face-centred cubic $(a=3.98$ $\AA$ ) lattice, ${ }^{3}$ we used the fragment $\mathrm{Tc}_{42}$, containing two metal layers. In this fragment the number of octahedral holes with diameter $3.98 \AA$ is 13 or $25 \%$ of Tc. Moreover, 24 unoccupied tetrahedral sites have a smaller diameter. Regular carbon allocation in accordance with the stoichiometry was realised as $\mathrm{Tc}_{42} \mathrm{C}_{7}$ (Figure 1). The model size was completely satisfied for this task, as the relative limiting number of placing carbon atoms exceeded the expecting value of 1:6.

At present, unfortunately, straight energy calculations for such a size of Tc compounds are not reliable due to computing difficulties and theory errors. For diatomic molecules, however, the stability may be estimated by the Mulliken bond overlap population index ${ }^{7} P$. For more complex compounds, the sum of these indexes $\Sigma P$ by overall bonds may be used. To get comparable results for different sizes and contents, it should be better to use a value related to one atom $P_{a}=\Sigma P / N_{a}$. For the model carbide, the stability may be found as a difference in $\Delta P$ between carbide $P_{a}(M C)$ and pure metal $P_{a}(M)$ indexes:

*Corresponding author. E-mail: plekhanov@ipc.rssi.ru. FAX: +7-095-335-17-38.

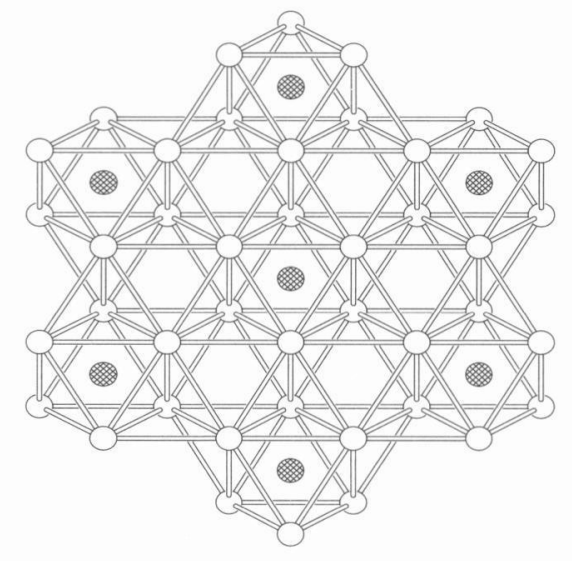

Figure 1. A model for technetium carbide cluster.

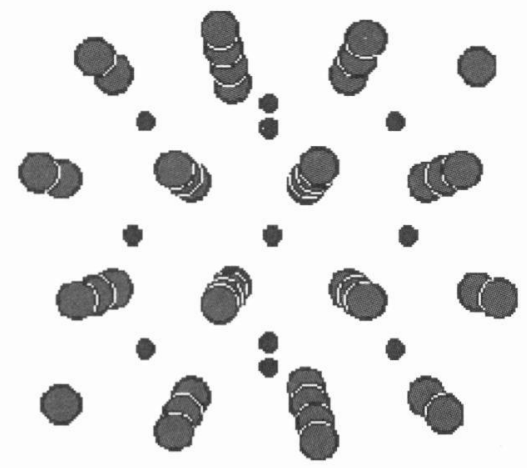

Figure 2. A model for $\alpha$-molybdenum carbide cluster.

$$
\Delta P=\left[(\Sigma P(T c T c)+\Sigma P(T c C)) /\left(N_{T_{c}}+N_{C}\right)\right]-\left[(\Sigma P(T c T c)) / N_{T c}\right] .
$$

To verify the $\Delta P$-criterion, we carried out calculations similar to the case of Mo carbide as the nearest Tc neighbour. The most stable carbide $\alpha-\mathrm{Mo}_{2} \mathrm{C}$ (Figure 2) crystallizes in the orthorhombic lattice. ${ }^{8}$ Octahedral hole $(4.195 \AA)$ sites in this lattice are half occupied by carbon regularly. In the model crystal with several tens of atoms but without surface $\mathrm{C}$-atoms, the stoichiometry must be greater than 2:1. The $\mathrm{Mo}_{44} \mathrm{C}_{x}$ fragment contains 4 metal layers. There are 17 octahedral holes in this model crystal. The real carbide composition corresponds to $x=11(25 \%)$. The $\Delta P$ values (Figure 3 ) are not quite regular row because the small size of model crystal and the small number of trial carbon positions. The minimum area at $\mathrm{C} / \mathrm{Mo}=$ $16 \%$ (or $33 \%$ for crystal) should be defined more exactly using expanded model crystals. Nevertheless, these data show that the $\Delta P$ tendency is fairly good. The stable composition corresponds to $\Delta P>0$. The maximum $\mathrm{C}$ : Mo value is slightly 


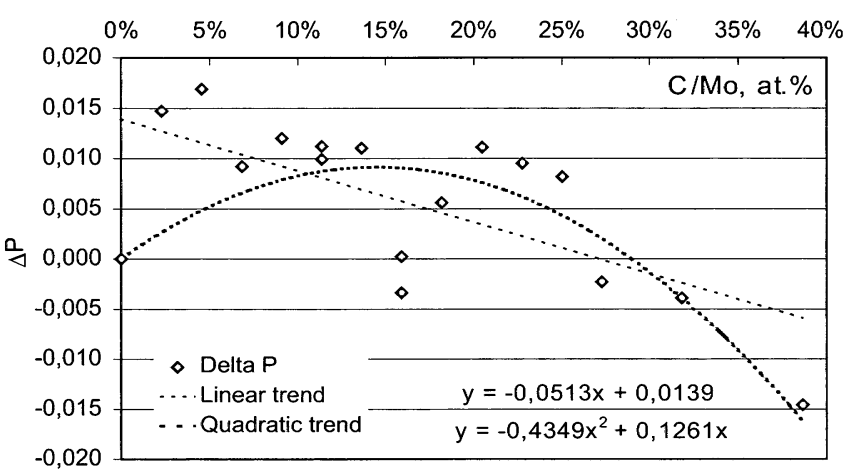

Figure 3. Bond overlap population index for a model molybdenum carbide.

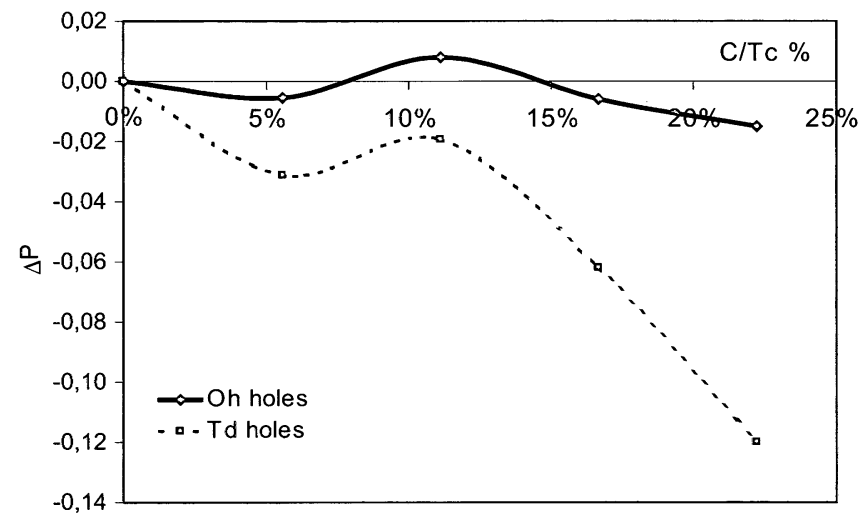

Figure 4. Bond overlap population index for octahedral and tetrahedral holes in a model technetium carbide.

greater than $25 \%$. On the contrary, the metal body-centred lattice hole filling is accompanied by $\Delta P$ decreasing, and in fact this carbide does not exist.

The good estimation for accepted criterion of a $\mathrm{Mo}_{2} \mathrm{C}$ composition allows us to apply it for the model Tc-carbide $\mathrm{Tc}_{42} \mathrm{C}_{X}$. Analysis of different coordination hole filling by this criterion shows that octahedral carbon position is much preferable (Figure 4). This is why we can use only octahedral carbon coordination models (Figure 1). Indeed, the tendency of $\Delta P$ changing (Figure 5) gives a stable area close to the experiment. The $\mathrm{C} / \mathrm{Tc}$ ratio does not exceed $15 \%$, and the $\mathrm{Tc}_{6} \mathrm{C}$ composition would be reasonable within the error limits of calculations ( as for $\mathrm{Mo}_{2} \mathrm{C}$ ).

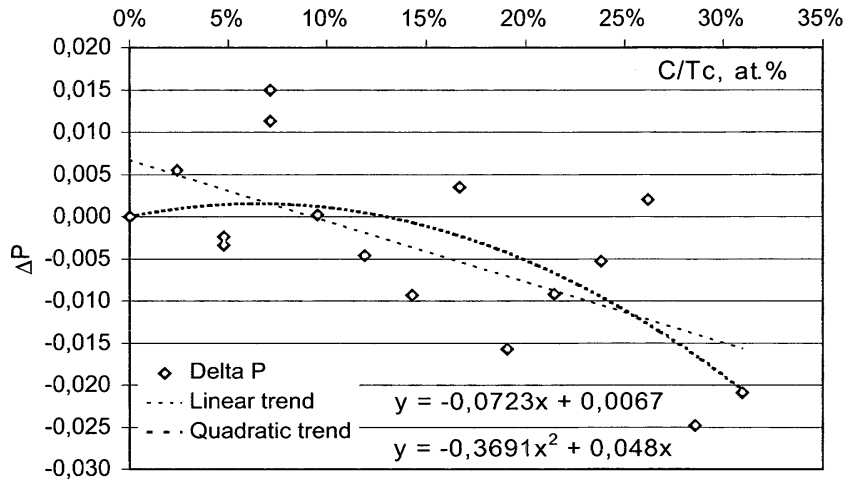

Figure 5. Bond overlap population index for a model technetium carbide.

On the other hand, the $\Delta P$ curve in the stable area contains sine-like parts. This region corresponds to local carbon concentration for more (maximum) and less (minimum) compound stability. It can be supposed that the alternate carbide phase formation is unregularly and unordered. It can included statistically averaged set of dissimilar fragments based on more stable local concentrations.

We can conclude that the $\Delta P$ criterion model is adequate for the stability estimation of rather complex compounds. The experimental Tc-carbide composition can be described by two possible models (regular and unregular) using the $\Delta P$ dependence.

\section{References}

(1) V. N. Eremenko, T. Ya. Velikanova, and A. A. Bondar, Poroshkovaya metalurgia (Russ) N11, 46 (1989).

(2) K. Guerman, T. Reich, C. Sergeant, R. Ortega, V. Tarasov, M. Simonoff, and G. Simonoff, Proceedings of OECD/NEA Workshop on pyrochemistry. Palais-des-Papes (Avignon, France) 14-16 March 2000.

(3) Yu. V. Plekhanov, K. E. German, and R. Sekine, Radiochem. (Russ.) 45, 217 (2003).

(4) H. Adachi, M. Tsukada, and C. Satoko, J. Phys. Soc. Jpn. 45, 875 (1978).

(5) J. C. Slater, Adv. Quant. Chem. 6, 1 (1972).

(6) R. S. Mulliken, Ann. Rev. Phys. Chem. 29, 1 (1978).

(7) J. Onoe et al, J. Phys. Soc. Jpn. 65, 2459 (1996).

(8) E. Parthe E. and V. Sadagopan, Acta Crystallographica 16, 202 (1963). 